\title{
Nanodiamonds with silicon vacancy defects for non-toxic photostable fluorescent labeling of neural precursor cells
}

\author{
Tobias D. Merson, ${ }^{1,2,3, *}$ Stefania Castelletto, ${ }^{4, *}$ Igor Aharonovich, ${ }^{5}$ Alisa Turbic, ${ }^{3}$ \\ Trevor J. Kilpatrick, ${ }^{1,2,3}$ Ann M. Turnley ${ }^{3}$ \\ ${ }^{1 .}$ Florey Institute of Neuroscience and Mental Health, \& Florey Department of Neuroscience and Mental Health, \\ Kenneth Myer Building, The University of Melbourne, Parkville, 3010, Victoria, Australia \\ 2. Melbourne Neuroscience Institute, The University of Melbourne, Parkville 3010, Victoria, Australia \\ 3. Centre for Neuroscience Research, Department of Anatomy and Neuroscience, The University of Melbourne, \\ Parkville, Victoria 3010, Australia \\ 4. School of Aerospace, Mechanical and Manufacturing Engineering RMIT University, Melbourne, Victoria 3000, Australia \\ 5. School of Physics and Advanced Materials, University of Technology Sydney, Broadway, NSW 2007, Australia \\ *Corresponding authors: tmerson@unimelb.edu.au \& stefaniacastelletto@gmail.com \\ Received Month X, XXXX; revised Month X, XXXX; accepted Month X, \\ XXXX; posted Month X, XXXX (Doc. ID XXXXX); published Month X, XXXX \\ Nanodiamonds (NDs) containing silicon vacancy (SiV) defects were evaluated as a potential biomarker for the labeling and \\ fluorescent imaging of neural precursor cells (NPCs). SiV-containing NDs were synthesized using chemical vapor deposition \\ followed by silicon atom irradiation. Spectrally, SiV-containing NDs exhibited extremely stable fluorescence and narrow \\ bandwidth emission with an excellent signal to noise ratio exceeding that of NDs containing nitrogen-vacancy (NV) centers. \\ NPCs labeled with NDs exhibited normal cell viability, metabolic and proliferative properties consistent with \\ biocompatibility. We conclude that SiV-containing NDs are a promising biomedical research tool for cellular labeling and \\ optical imaging in stem cell research. (C) 2013 Optical Society of America \\ OCIS Codes: (160.2540) Fluorescent and luminescent materials, (160.4236) Nanomaterials, (170.3880) Medical and \\ biological imaging, (170.1530) Cell analysis, (260.1180) Crystal optics. \\ http://dx.doi.org/10.1364/OL.99.099999
}

Fluorescent biomarkers for labeling cellular and molecular targets are emerging as increasingly important tools in biomedical research and modern medicine. The range of potential applications is diverse, from monitoring drug or tumor localization within the body [1], to assessing the migration of transplanted stem cells used in cell based therapies [2,3].

A major goal is to develop improved fluorescent labeling reagents that achieve optimal fluorescence intensity without photo-bleaching or blinking. These latter properties are observed with most currently used fluorescent proteins, quantum dots, metallic and dielectric beads and hinders their use for long-term repeated imaging applications [4]. An additional goal is to generate fluorescent biomarkers that can be specifically targeted to distinct cellular or molecular targets via the conjugation of antibodies, growth factors, organic chemicals or drugs.

In these respects, nanodiamonds (NDs) offer several advantages. First, atomic changes in ND surface structure produces bright optical defects that possess unrivalled photostability, even for the smallest NDs $\left(\sim 5^{-}\right.$ $10 \mathrm{~nm})[5,6]$. Second, being made of carbon, they are biocompatible, non-toxic and highly amenable to surface functionalization, enabling the conjugation of various biomolecules such as DNA, siRNA and proteins [7-10].

Despite these promising features, further improvement of the optical properties of NDs could enhance their utility as fluorescent biomarkers. The most common optical defect that can be "naturally" incorporated into the NDs is a nitrogen vacancy center (NV), a nitrogen atom close to a vacancy in the diamond lattice [11]. NV emits over a broad range of wavelengths in the visible spectrum, and has been explored as a potential candidate for atomic resolution magnetic resonance imaging. However despite its promising properties for magnetic sensing [12], the optical properties of NV centers are not ideal. Specifically, the emission wavelengths of the NV are very broad (638 $\mathrm{nm}$ to $750 \mathrm{~nm}$ ), its peak absorption at $532 \mathrm{~nm}$ overlaps with wavelengths that excite cellular auto-fluorescence and the defect also possesses a long excited state lifetime of $22 \mathrm{~ns}$ that hinders its efficiency. By contrast, NDs containing silicon vacancy $(\mathrm{SiV})$ defects could offer optimal spectral properties for cellular imaging.

In this Letter, we report on the generation of ultrabright NDs hosting SiV defects and their use as effective biomarkers of primary neural precursor cells (NPCs) isolated from the adult mouse brain. We compare the optical properties and biocompatibility of NDs incorporating $\mathrm{NV}$ versus $\mathrm{SiV}$ defects and demonstrate the utility of the latter for cellular imaging.

The spectral properties of SiV-containing NDs make them ideal candidates for fluorescent biomarkers. The SiV defects consist of a silicon atom in the diamond lattice taking the place of 2 carbon vacancies [13]. The SiV 
possesses a very narrow fluorescence bandwidth $(\sim 4 \mathrm{~nm})$ centered at $740 \mathrm{~nm}$, its excited state lifetime is extremely short ( $1 \mathrm{~ns})$ enabling fast data acquisition and tracking and the centers can be observed in ultra small NDs (sub 5 nm, Ref. 5) [14,15]. Furthermore, since the emission is fully polarized, this in principle allows emission to be tracked by polarization measurements or dipole imaging. The combination of these properties makes NDs hosting the $\mathrm{SiV}$ defects a prime candidate as an efficient biolabeling reagent.

To generate fluorescent NDs based on SiV defects, we used two different methods. For Method 1, NDs were grown on a fixed silicon substrate using 4-6 nm seeding NDs to nucleate growth in a microwave plasma chemical vapor deposition (CVD) reactor (Astex) at $900^{\circ} \mathrm{C}, 150$ Torr and $950 \mathrm{~W}$ followed by annealing for $2 \mathrm{hrs}$ at $1000^{\circ} \mathrm{C}$ in forming gas. This achieves a growth rate of approximately $28 \mathrm{~nm} / \mathrm{min}$ resulting in the production of NDs with an average size of $200 \mathrm{~nm}$ after $8 \mathrm{~min}$ [16]. Characterization of these NDs by scanning electron microscopy (SEM) revealed uniform crystalline structures (Fig. 1(a)). Due to the fast growth rate the incorporation of silicon from the etched substrate is considerable and strong fluorescence peaking at $738 \mathrm{~nm}$ dominated the spectra. In Fig. 1(b) a typical PL spectra recorded from the NDs at room temperature using a $532 \mathrm{~nm}$ excitation is shown. A sharp peak at $738 \mathrm{~nm}$ is the zero phonon line (ZPL) of the SiV. A comparison of the emission spectra of NV-containing NDs is shown.

Fig 1(c) demonstrates the exceptional photostability of fluorescent light emission from excited SiV centers with little to no evidence of fluorescent quenching over a period of $96 \mathrm{~min}$ of continuous excitation. Very few naturallyoccuring NV centers were found in the seed crystal. As a result, grown NDs consist of much higher concentrations of SiV compared to NV.

The second approach to generate $\mathrm{SiV}$-containing NDs (Method 2) was devised to ensure NDs remained in a powder form so that they could be added to cells grown in suspension culture. To achieve this, we utilized an ion accelerator to implant $\mathrm{Si}$ into untreated $80 \mathrm{~nm}$ high pressure high temperature (HPHT) NDs (1017 Si ions $/ \mathrm{cm}^{2}$ ) at room temperature. After implantation, NDs were annealed for $1 \mathrm{hr}$ in forming gas to create the defects and remove residual graphite from the implantation. No acid cleaning was performed therefore the fluorescence emission was not optimized.

Compared to the first approach, Method 2 resulted in a much lower yield of SiV defects per ND, but had the distinct advantage that NDs remained in powder form so that they could be added to NPCs grown in suspension culture whereas NDs generated using Method 1 required NPCs to be grown as an adherent monolayer on top of the silicon substrate.

To test the biocompatibility and fluorescent labeling properties of NDs, we established primary cultures of adult mouse NPCs grown as free-floating colonies of cells known as neurospheres. Primary neurospheres were derived from subventricular zone tissue isolated from the brains of four adult C57BL/6 mice that were humanely sacrificed at eight weeks of age (Fig. 2(a)), using procedures approved by the animal ethics committee of the Florey Institute of Neuroscience and Mental Health. Single cell suspensions of NPCs for cell assays or for serial passaging were generated by neurosphere dissociation every 5-7 days, using techniques described previously [17].

First we assessed whether labeling NPCs with NDs influenced cell proliferation and/or cell viability. NPCs were plated at a density of 50,000 cells/well in 24-well plates containing proliferation medium and 0, 20, 40 or

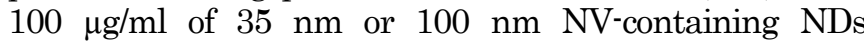
(triplicate wells were used for each condition). Fig. 2(b) reveals photomicrographs of typical NPC cultures grown either under basal conditions (without NDs) or with 100 $\mu \mathrm{g} / \mathrm{ml}$ of $35 \mathrm{~nm}$ or $100 \mathrm{~nm}$ NDs. NPC-derived neurospheres cultured in the presence of NDs were indistinguishable from NPCs under basal conditions. At concentrations of $100 \mu \mathrm{g} / \mathrm{ml}$, the uptake of $100 \mathrm{~nm}$ NDs into NPCs was evident under light microscopy resulting in a phase dark appearance of cells (Fig. 2(b)).

Plots in Fig. 2(c) reveal that the increase in NPC yield due to cell proliferation over 7 days in culture was unaffected by $100 \mathrm{~nm}$ NDs present at up to $40 \mu \mathrm{g} / \mathrm{ml}$. However, when presented in excess $(100 \mu \mathrm{g} / \mathrm{ml})$, NDs induced a small but statistically significant increase in cell yield relative to the basal condition.

We also measured the amount of lactate dehydrogenase $(\mathrm{LDH})$ released into the culture medium after 4 and 7 days culture, to determine whether NDs elicit NPC cytotoxicity. Fifty microliters of supernatant from each well was processed using the CytoTox 96® NonRadioactive Cytotoxicity Assay (Promega, Madison, WI, USA), according the manufacturer's instructions. Plots in Fig. 2(d) revealed that, relative to basal conditions, $100 \mathrm{~nm}$ NDs did not influence the level of $\mathrm{LDH}$ released into the medium of NPC cultures. Similar results were obtained with $35 \mathrm{~nm}$ NDs over the same range of concentrations (data not shown). Collectively, these data demonstrated that NDs had no effect on NPC viability or proliferation, with the exception that $100 \mathrm{~nm}$ NDs at $100 \mu \mathrm{g} / \mathrm{ml}$ induced a small increase in the yield of NPCs after 7 days culture.

Next we compared the fluorescence properties of $\mathrm{NV}$ and SiV centers for NDs bound or unbound to NPCs. NPCs were grown directly on silicon substrates studded with synthesized SiV NDs (generated by Method 1) or by suspension culture in the presence of $\mathrm{NV}^{-}$or $\mathrm{SiV}^{-}$ containing NDs (the latter generated by Method 2). For suspension cultures, NPCs were allowed to adhere to laminin-coated glass coverslips overnight then fixed with methanol $\left(-20^{\circ} \mathrm{C}\right.$ for 10 mins $)$ and mounted on a glass slide before imaging. NPCs grown on silicon were fixed in an identical manner. For NV-containing NDs, samples were excited using a $100 \mathrm{~mW}$ continuous wave laser operating at $532 \mathrm{~nm}$, with $50 \mathrm{\mu W}$ power incident on the sample. The laser was focused through the back surface of the coverslip and onto the sample using a $100 \mathrm{x}$ infinity corrected oil immersion objective lens with a numerical aperture of 1.3, and luminescence was collected confocally through a pinhole. A spectrometer with a cooled CCD (Princeton Instruments) was used to characterize the luminescence, single-photon-sensitive avalanche photodiodes (Perkin-Elmer SPCM-AQR-14) were used to measure the photon count rate for the confocal scan. A $650 \mathrm{~nm}$ long pass filter was used to selectively collect the 
photons from NV center. For imaging $\mathrm{SiV}$ in NDs we used a $690 \mathrm{~nm}$ CW laser as excitation in a similar confocal system, while a $740 \pm 10 \mathrm{~nm}$ bandpass filter was used to collect photons from the specific defects.

We initially incubated NPCs with $35 \mathrm{~nm}$ and $100 \mathrm{~nm}$ NDs containing high concentration of the nitrogen vacancy (NV) centers. Fig 3(b) shows epifluorescence imaging of NPCs grown for 7 days in the presence of NVcontaining NDs. The broad emission spectra overlapped significantly with intrinsic cellular auto-fluorescence, particularly for NDs of $35 \mathrm{~nm}$. Although the same imaging parameters were sufficient to detect a fluorescent signal from $100 \mathrm{~nm}$ NDs, the signal could not be completely separated from that elicited by cellular autofluorescence, resulting in low-contrast images.

To examine the spectral properties of SiV-containing NDs for cellular labeling, NPCs were grown for four days either as adherent cultures on Silicon substrates studded with $80 \mathrm{~nm}$ NDs grown by CVD or as suspension cultures with $40 \mathrm{~nm}$ NDs that were implanted with Si. In the first case, the $\mathrm{SiV}$ signal was restricted to the Silicon surface indicating limited internalization of substrate-bound NDs by NPCs (data not shown). By contrast, NDs containing $\mathrm{SiV}$ defects introduced by $\mathrm{Si}$ implantation labeled cells with great efficiency. Figure 4(a) shows a confocal image collected using an Olympus FV-1000 microscope of NPCs cultured with $40 \mathrm{~nm}$ SiV-implanted NDs. The pseudocolored fluorescent signal illustrates the range of fluorescent signal intensities detected among labeled cells. The fluorescent image is overlaid with a bright-field image of the cells collected using Nomarski optics. Fig. 4(b) shows the spectrum recorded from the NDs in a cell culture, and outside the cell. The $\mathrm{SiV}$ emission is preserved and the cell does not modify the $\mathrm{SiV}$ emission. A comparison with CVD SiV in ND spectra is also shown. The SiV containing NDs offer a superior cell labeling, with very bright photo-stable fluorescence, broad absorption spectra and narrow emission spectra at the near infra red that does not overlap with cellular auto-fluorescence.

Our data reveal that the optimal spectral properties of SiV-containing NDs make them excellent candidates for ongoing development as cellular biomarkers. The development of techniques to mass produce NDs with $\mathrm{SiV}$ centers is therefore an important consideration. This can be achieved via CVD growth and successive etching of the sacrificial substrate and mechanical milling [15], rather than with ion implantation. By comparison, the mass production of enhanced fluorescent NDs based on NV centers is likely more cumbersome due to the need to use electron irradiation, which not easily available. Our study highlights the value in investigating the suitability of NDs containing other color centers for spectral imaging. In particular, NDs with Cr-related centers have similar spectral properties to $\mathrm{SiV}$ [19], however mass production of NDs with Cr-related centers is likely to be more problematic than for $\mathrm{SiV}$ centers.

In summary, we demonstrate that SiV-containing NDs are excellent biomarkers that have highly favorable attributes compared to NV-containing NDs. Specifically, SiV-containing NDs exhibit bright, photostable emission with a broad absorption spectra and narrow emission spectra in the near infrared range which bypasses issues of cell auto-fluorescence. SiV-containing NDs prove to be ideal candidates for cell labeling since they exhibit very bright and photo-stable fluorescence, In comparison, NDs containing a nitrogen vacancy complex (NV center) are unsuitable for cellular labeling due to their narrow absorption spectra and broad emission spectra which overlaps with intrinsic cellular auto-fluorescence.

In conclusion, the exceptional spectral properties of SiVcontaining NDs including narrow line-width in the near infrared, short excited state lifetime and excellent photostability, make them very attractive for biological applications. Further exploration of the utility of SiVcontaining NDs for cellular imaging could provide promising avenues for the development of high resolution non-invasive real-time imaging of cell migration in vivo.

This project was supported by the University of Melbourne interdisciplinary research grant scheme.

\section{References}

1. Y. Urano, Curr. Opin. Chem. Biol. 16, 602 (2012).

2. M. Modo, Curr. Opin. Organ Transplant. 13, 654 (2008).

3. E. Gu, W.-Y. Chen, J. Gu, P. Burridge, and J. C. Wu, Theranostics 2, 335 (2012).

4. J. Vogelsang, R. Kasper, C. Steinhauer, B. Person, M. Heilemann, M. Sauer, and P. Tinnefeld, Angew. Chem. Int. Ed. Engl. 47, 5465 (2008).

5. I. I. Vlasov, A. S. Barnard, V. G. Ralchenko, O. I. Lebedev, M. V. Kanzyuba, A. V. Saveliev, V. I. Konov, and E. Goovaerts, Adv. Mater. 21, 808 (2009).

6. I. I. Vlasov, O. Shenderova, S. Turner, O. I. Lebedev, A. A. Basov, I. Sildos, M. Rähn, A. A. Shiryaev, and G. Van Tendeloo, Small 6, 687 (2010).

7. X. -Q. Zhang, M. Chen, R. Lam, X. Xu, E. Osawa, and D. Ho, ACS Nano 3, 2609 (2009).

8. N. Mohan, C. -S. Chen, H. -H. Hsieh, Y. -C. Wu, and H. -C. Chang, Nano Lett. 10, 3692 (2010).

9. V. N. Mochalin, O. Shenderova, D. Ho, and Y. Gogotsi, Nature Nanotech. 7, 11 (2011).

10. Krueger and D. Lang, Adv. Funct. Mater. 22, 890 (2012).

11. G. Davies and M. F. Hamer, Proc. R. Soc. Lond. A Math. Phys. Sci. 348, 285 (1976).

12. D. Le Sage, K. Arai, D. R. Glenn, S. J. DeVience, L. M. Pham, L. Rahn-Lee, M.D. Lukin, A. Yacoby, A. Komeili, and R.L. Walsworth, Nature 496, 486 (2013).

13. J. P. Goss, R. Jones, S. J. Breuer, P. R. Briddon, and S. Öberg, Phys. Rev. Lett. 77, 3041 (1996).

14. E. Neu, D. Steinmetz, J. Riedrich-Möller, S. Gsell, M. Fischer, M. Schreck, and C. Becher, New J. Phys. 13, 025012 (2011).

15. E. Neu, M. Fischer, S. Gsell, M. Schreck, and C. Becher, Phys. Rev. B 84, 205211 (2011).

16. Stacey, I. Aharonovich, S. Prawer, and J.E. Butler, Diam. Relat. Mater. 18, 51 (2009).

17. T. D. Merson, M. P. Dixon, C. Collin, R. L. Rietze, P. F. Bartlett, T. Thomas, and A. K. Voss, J. Neurosci. 26, 11359 (2006). 
18. I. Aharonovich, S. Castelletto, B.C. Johnson, J.C. McCallum, and S. Prawer, New J. Phys. 13, 045015 (2011). (a)

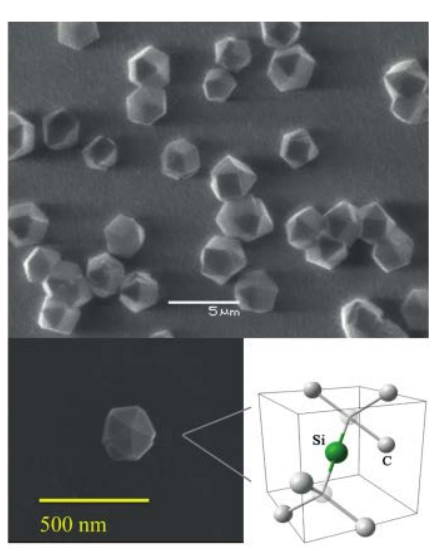

(b)

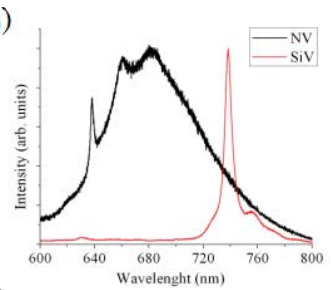

(c)

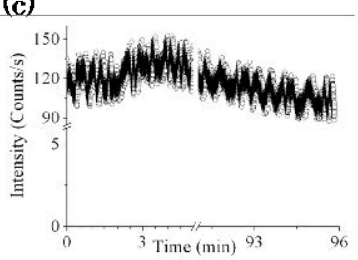

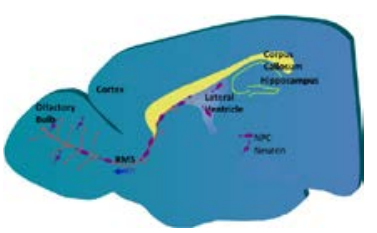

(d)

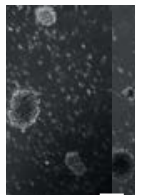

ay $4 \quad$ Day 7

Fig. 2. NPCs isolated from the adult mouse brain exhibit high biocompatibility with NDs. (a) Schematic view of the adult mouse brain in sagittal axis. Primary NPCs were isolated from the subventricular zone of the lateral ventricles. NPCs contribute to neurogenesis by migrating to the olfactory bulb via the rostral migratory stream (RMS). (b) Morphology of NPC-derived neurosphere cultures assessed 7 days after culture with NDs added at time of passaging. Scale bar: $100 \mu \mathrm{m}$. (c) NPCs labeled with 20 or $40 \mu \mathrm{g} / \mathrm{ml}$ NDs exhibited normal increases in cell yield. NDs at $100 \mu \mathrm{g} / \mathrm{ml}$ caused a small but significant increase in cell yield relative to basal $\left({ }^{*} \mathrm{P}<0.05\right.$, one-way ANOVA, Tukey post-hoc analysis). (d) Assessment of cell death of NPCs cultured in the presence of NDs using the LDH cytotoxicity assay. After 4 and 7 days of culture NPCs cultured with NDs had LDH levels that were equivalent to those cultured under basal conditions without NDs indicating that NDs are biocompatible and non-toxic. (a)

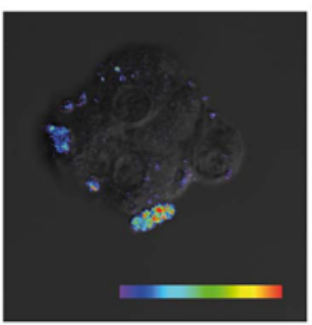

(b)

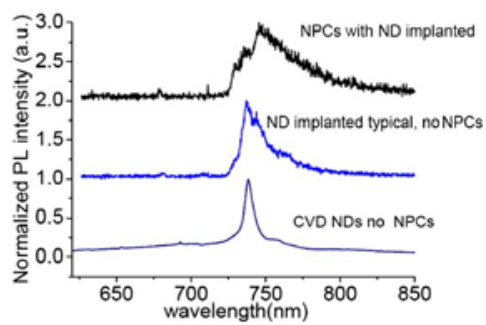

Fig. 3. (a) High power confocal micrograph of a single NPC labeled with NV-containing NDs reveals significant intracellular uptake (b) Epifluorescence image of NSCs grown with enhanced NV centres containing NDs (upper images) and dark field images (bottom) of the same sample area. The NDs incubated with the cells were 35 and 100 $\mathrm{nm}$ respectively. The $35 \mathrm{~nm}$ NDs show a limited emission compared to the $100 \mathrm{~nm}$.

Fig. 4. (a) (b) A confocal image of NPCs grown on NDs merged with a bright field image of the neurosphere in grayscale revealing the exceptional contrast of SiVcontaining NDs over cell background. (b) PL of SiV in CVD NDs and NDs implanted with Si in cell culture and without cells. The broader emission of the $\mathrm{SiV}$ in the cultured cell sample was attributed to the use of a thick cover glass for which the objective was not fully corrected, inducing a larger focal volume collection compared to the case where only NDs were imaged. 


\section{Full Citation Listings}

1. Urano, Y., "Novel live imaging techniques of cellular functions and in vivo tumors based on precise design of small molecule-based "activatable” fluorescence probes," Curr Opin Chem Biol 16, 602-608 (2012).

2. Modo, M., "Noninvasive imaging of transplanted cells," Curr Opin Organ Transplant 13, 654-658 (2008).

3. Gu, E., Chen, W.-Y., Gu, J., Burridge, P., and Wu, J. C., "Molecular imaging of stem cells: tracking survival, biodistribution, tumorigenicity, and immunogenicity," Theranostics 2, 335-345 (2012).

4. Vogelsang, J., Kasper, R., Steinhauer, C., Person, B., Heilemann, M., Sauer, M., and Tinnefeld, P., "A reducing and oxidizing system minimizes photobleaching and blinking of fluorescent dyes," Angew. Chem. Int. Ed. Engl. 47, 5465-5469 (2008).

5. Vlasov, I. I., Barnard, A. S., Ralchenko, V. G., Lebedev, O. I., Kanzyuba, M. V., Saveliev, A. V., Konov, V. I., and Goovaerts, E., "Nanodiamond Photoemitters Based on Strong Narrow-Band Luminescence from Silicon-Vacancy Defects," Adv Mater 21, 808-812 (2009).

6. Vlasov, I. I., Shenderova, O., Turner, S., Lebedev, O. I., Basov, A. A., Sildos, I., Rähn, M., Shiryaev, A. A., and Van Tendeloo, G., "Nitrogen and luminescent nitrogen-vacancy defects in detonation nanodiamond," Small 6, 687-694 (2010).

7. Zhang, X.-Q., Chen, M., Lam, R., Xu, X., Osawa, E., and Ho, D., "Polymer-functionalized nanodiamond platforms as vehicles for gene delivery," ACS Nano 3, 2609-2616 (2009).

8. Mohan, N., Chen, C.-S., Hsieh, H.-H., Wu, Y.-C., and Chang, H.-C., "In vivo imaging and toxicity assessments of fluorescent nanodiamonds in Caenorhabditis elegans," Nano Letters 10, 3692-3699 (2010).

9. Mochalin, V. N., Shenderova, O., Ho, D., and Gogotsi, Y., "The properties and applications of nanodiamonds," Nature Nanotechnology 7, 11-23 (2011).

10. Krueger, A. and Lang, D., "Functionality is key: recent progress in the surface modification of nanodiamond," Advanced Functional Materials 22, 890-906 (2012).

11. Davies, G. and Hamer, M. F., "Optical studies of the $1.945 \mathrm{eV}$ vibronic band in diamond," Proceedings of the Royal Society of London. A. Mathematical and Physical Sciences 348, 285-298 (1976).

12. Le Sage, D., Arai, K., Glenn, D. R., DeVience, S. J., Pham, L. M., Rahn-Lee, L., Lukin, M. D., Yacoby, A., Komeili, A., and Walsworth, R. L., "Optical magnetic imaging of living cells," Nature 496, 486-489 (2013).
13. Goss, J. P., Jones, R., Breuer, S. J., Briddon, P. R., and Öberg, S., "The twelve-line 1.682 eV luminescence center in diamond and the vacancy-silicon complex," Physical Review Letters 77, 3041 (1996).

14. Neu, E., Steinmetz, D., Riedrich-Möller, J., Gsell, S., Fischer, M., Schreck, M., and Becher, C., "Single photon emission from silicon-vacancy colour centres in chemical vapour deposition nano-diamonds on iridium," New Journal of Physics 13, 025012 (2011).

15. Neu, E., Fischer, M., Gsell, S., Schreck, M., and Becher, C., "Fluorescence and polarization spectroscopy of single silicon vacancy centers in heteroepitaxial nanodiamonds on iridium," Phys. Rev. B 84, 205211 (2011).

16. Stacey, A., Aharonovich, I., Prawer, S., and Butler, J. E., "Controlled synthesis of high quality micro/nano-diamonds by microwave plasma chemical vapor deposition," Diamond and Related Materials 18, 51-55 (2009).

17. Merson, T. D., Dixon, M. P., Collin, C., Rietze, R. L., Bartlett, P. F., Thomas, T., and Voss, A. K., "The transcriptional coactivator Querkopf controls adult neurogenesis," Journal of Neuroscience 26, 11359-11370 (2006).

18. Aharonovich, I., Castelletto, S., Johnson, B. C., McCallum, J. C., and Prawer, S., "Engineering chromiumrelated single photon emitters in single crystal diamonds," New Journal of Physics 13, 045015 (2011). 